\title{
EXISTENCE AND UNIQUENESS RESULTS FOR A NONLINEAR STATIONARY SYSTEM
}

\author{
OLIVIER GUIBÉ \\ Laboratoire de Mathématiques Raphaël Salem \\ UMR CNRS 6085, Site Colbert \\ Université de Rouen \\ F-76821 Mont Saint Aignan cedex \\ E-mail : olivier.guibe@univ-rouen.fr
}

\begin{abstract}
We prove a few existence results of a solution for a static system with a coupling of thermoviscoelastic type. As this system involves $L^{1}$ coupling terms we use the techniques of renormalized solutions for elliptic equations with $L^{1}$ data. We also prove partial uniqueness results.
\end{abstract}

\section{INTRODUCTION}

In the present paper we consider the following nonlinear coupled system:

$$
\begin{array}{ll}
\lambda u-\operatorname{div}(\mathbf{A}(x) D u-f(\theta))=g & \text { in } \Omega, \\
\mu \theta-\operatorname{div}(\mathbf{a}(x, D \theta))=(\mathbf{A}(x) D u-f(\theta)) \cdot D u & \text { in } \Omega, \\
u=0 \quad \theta=0 & \text { on } \partial \Omega,
\end{array}
$$

where $\Omega$ is an open and bounded subset of $\mathbb{R}^{N}(N \geq 2), \lambda, \mu>0, f: \mathbb{R} \mapsto \mathbb{R}^{N}$ is a continuous function, $g \in L^{2}(\Omega), \mathbf{A}(x)$ is a coercive matrix with $L^{\infty}$-coefficients and $v \mapsto-\operatorname{div}(\mathbf{a}(x, D v))$ is a monotone operator defined from $H_{0}^{1}(\Omega)$ into $H^{-1}(\Omega)$.

Problem (11)-(3) is a static version (or time discretized-version) of a time dependent class of systems in thermoviscoelasticity. Under stronger assumptions than in the present paper, existence of a solution for these evolution systems is established in [4] (see also [3]). Moreover, for (1) -(3) uniqueness results were also proved.

The main difficulties in dealing with existence of solution of system (11)-(3) are due to equation (2) and the coupling. Indeed if $u$ is a variational solution of (11) (i.e. $u \in H_{0}^{1}(\Omega)$ ) then the right-hand side of (2) belongs to $L^{1}(\Omega)$. It follows from L. Boccardo and T. Gallouët 6] (see also [2] and [20]) that $\theta$ is expected in $L^{q}(\Omega)$ for $q<N /(N-2)$ if $N \geq 3$ and $q<\infty$ if $N=2$. With the aim of solving (11) with $f(\theta) \in L^{2}(\Omega)$ we are then led to assume that $f$ satisfies the growth assumption

$$
\forall r \in \mathbb{R} \quad|f(r)| \leq a+M|r|^{\alpha},
$$

with $a>0, M>0$ and $\alpha<N /(2(N-2))$ if $N \leq 3$ and $\alpha<\infty$ if $N=2$. Under this hypothesis on $f$, the coupling between Equations (11) and (2) together with the L. Boccardo

Key words and phrases. nonlinear coupled system, renormalized solutions, $L^{1}$ data. 
and T. Gallouët estimates techniques (see [6] and Remark 5.5 of the present paper) lead to the following a priori estimate on $\theta$,

$$
\forall q \in\left[1, \frac{N}{N-2}\left[\quad\|\theta\|_{L^{q}(\Omega)} \leq C\left(1+\|f(\theta)\|_{L^{2}(\Omega)}^{2}\right) \leq C^{\prime}\left(1+\int_{\Omega}|\theta|^{2 \alpha} \mathrm{d} x\right),\right.\right.
$$

where $C$ and $C^{\prime}$ are real positive constant independ of $u$ and $\theta$. This implies that if $2 \alpha \geq 1$ the estimate above is not sufficient in general settings to obtain the existence of a solution of (11) - (3) using a fixed-point or approximation method.

As the right-hand side of (2) belongs to $L^{1}$, we use in the present paper the convenient framework of renormalized solutions that insures uniqueness and stability results for equations with $L^{1}$ data. Renormalized solutions have been introduced by R.J. DiPerna and P.-L. Lions in [11] and [12] for first order equations and have been adapted for elliptic equations in [5], [18, [19] and for elliptic equations with general measure data in [9] (see also [8]). Other frameworks as entropy solutions [2] or SOLA [10] may be used for equation (2) with $L^{1}$ data.

Another interesting question related to problem (11)-(3) deals with the uniqueness of a solution, that is an open problem in general settings due to lack of regularity of $\theta$ and the right-hand side of (21). We investigate in the present paper uniqueness of a small solution $(u, \theta)$ such that $\theta \geq 0$ almost everywhere in $\Omega$ and under additional assumptions on the data for $N=2$ and $N=3$.

Elliptic systems involving $L^{1}$ coupling terms are also studied in [13], 7] and [17] and use a convenient formulation for equation with the $L^{1}$ term.

The plan of this paper is as follows. In Section 2 we recall the definition of a renormalized solution and we define a weak-renormalized solution for system (11)-(3). In Section 3 we give a few useful properties on renormalized solutions. Section 4 and Section 5 are devoted to existence results for two restricted case: the first case deals with small data, the second case contains existence results under more restrictive conditions on $f$ but for general data. Section 6 contains a partial uniqueness result of a small solution $(u, \theta)$ such that $\theta \geq 0$ almost everywhere in $\Omega$ and under additional assumptions on the data.

\section{Assumptions And Definitions}

Let $\Omega$ be an open and bounded subset of $\mathbb{R}^{N}(N \geq 2)$. The following assumptions are made on the data:

(A1) $\mathbf{A}(x)$ is a coercive matrix field with coefficients lying in $L^{\infty}(\Omega)$ i.e. $\mathbf{A}(x)=\left(a_{i, j}(x)\right)_{1 \leq i, j \leq N}$ with

- $a_{i, j}(x) \in L^{\infty}(\Omega)$

- $\exists \gamma>0$ such that $\forall \xi \in \mathbb{R}^{N} \mathbf{A}(x) \xi \cdot \xi \geq \gamma\|\xi\|_{\mathbb{R}^{N}}^{2} \quad$ for almost every $x \in \Omega$;

(A2) the function a : $\Omega \times \mathbb{R}^{N} \longmapsto \mathbb{R}^{N}$ is a Caratheodory function (continuous in $\xi$ for almost every $x \in \Omega$ and measurable in $x$ for every $\xi \in \mathbb{R}^{N}$ ) and there exists $\delta>0$ such that

$$
\forall \xi \in \mathbb{R}^{N} \quad \mathbf{a}(x, \xi) \cdot \xi \geq \delta\|\xi\|_{\mathbb{R}^{N}}^{2} \quad \text { for almost every } x \in \Omega ;
$$

(A3) for every $\xi$ and $\xi^{\prime}$ in $\mathbb{R}^{N}$, and almost everywhere in $\Omega$

$$
\left(\mathbf{a}(x, \xi)-\mathbf{a}\left(x, \xi^{\prime}\right)\right) \cdot\left(\xi-\xi^{\prime}\right) \geq 0 ;
$$

(A4) there exists $\beta>0$ such that

$$
|\mathbf{a}(x, \xi)| \leq \beta(b(x)+|\xi|)
$$


holds for every $\xi \in \mathbb{R}^{N}$ and for almost every in $x \in \Omega$ with $b \in L^{2}(\Omega)$;

(A5) $\lambda>0, \mu>0$

(A6) $f$ is a continuous function defined on $\mathbb{R}$ with values in $\mathbb{R}^{N}$;

(A7) $g$ is an element of $L^{2}(\Omega)$.

Throughout this paper and for any non negative real number $K$ we denote by $T_{K}(r)$ the truncation function at height $\pm K$, i.e. $T_{K}(r)=\min (K, \max (r,-K))$. For a measurable set $E$ of $\Omega$, we denote by $\mathbb{1}_{E}$ the characteristic function of $E$.

Following [18] (and [19]) we recall the definition of a renormalized solution for nonlinear equations of type (2) with $L^{1}$ right-hand side.

Definition 2.1. Let $F$ be an element of $L^{1}(\Omega)$. A measurable function $\theta$ defined on $\Omega$ is called a renormalized solution of the problem

$$
P(F)\left\{\begin{array}{l}
\mu \theta-\operatorname{div}(\mathbf{a}(x, D \theta))=F \quad \text { in } \Omega, \\
\theta=0 \text { on } \partial \Omega
\end{array}\right.
$$

if

$$
\theta \in L^{1}(\Omega), \forall K>0 \quad T_{K}(\theta) \in H_{0}^{1}(\Omega)
$$

for every function $h \in W^{1, \infty}(\mathbb{R})$ such that $h$ has a compact support,

$$
\begin{aligned}
& \mu \theta h(\theta)-\operatorname{div}(h(\theta) \mathbf{a}(x, D \theta))+h^{\prime}(\theta) \mathbf{a}(x, D \theta) \cdot D \theta=F h(\theta) \text { in } \mathcal{D}^{\prime}(\Omega) ; \\
& \lim _{n \rightarrow \infty} \frac{1}{n} \int_{\{n<|\theta|<2 n\}}|D \theta|^{2} \mathrm{~d} x=0 .
\end{aligned}
$$

Under assumptions (A2) - (A4) and with $\mu>0$, using the techniques developped in [18] (see also [9] and [19]), there exists a unique renormalized solution of $P(F)$ for any $F$ in $L^{1}(\Omega)$.

We now use renormalized solutions to define a so called weak-renormalized solution of Problem (1)-(3).

Definition 2.2. A couple of functions $(u, \theta)$ defined on $\Omega$ is called a weak-renormalized solution of (11)-(3) if $u$ and $\theta$ satisfy

$$
\begin{gathered}
u \in H_{0}^{1}(\Omega), \\
f(\theta) \in\left(L^{2}(\theta)\right)^{N}, \\
\lambda u-\operatorname{div}(\mathbf{A}(x) D u-f(\theta))=g \text { in } \mathcal{D}^{\prime}(\Omega), \\
\theta \text { is a renormalized solution of (22)-(3). }
\end{gathered}
$$

Under regularities (7)-(8), the right-hand side of (2), $(\mathbf{A}(x) D u-f(\theta)) \cdot D u$, belongs to $L^{1}(\theta)$. So we are in the framework of renormalized solution for equation (2).

\section{USEFUL PROPERTIES OF RENORMALIZED SOLUTIONS}

We recall the following propositions on renormalized solutions of elliptic equations for $L^{1}$ data, that can be shown using the techniques developped in [9], [18] and [19].

Proposition 3.1 (Existence and uniqueness of the renormalized solution). Assume that (A2) - (A4) hold true and $\mu>0$. Then for any $F$ belonging to $L^{1}(\Omega)$, there exists a unique 
renormalized solution of Problem $P(F)$. Moreover for any function $w \in L^{\infty}(\Omega) \cap H_{0}^{1}(\Omega)$, if there exists $K>0$ such that $D w=0$ almost everywhere in $\{x:|\theta(x)| \geq K\}$ then

$$
\mu \int_{\Omega} \theta w \mathrm{~d} x+\int_{\Omega} \mathbf{a}(x, D \theta) \cdot D w \mathrm{~d} x=\int_{\Omega} F w \mathrm{~d} x .
$$

Remark 3.2. Equality (11) which is proved in [9] in the context of general measure data, is formally obtained through using the test function $w$ in the equation of $P(F)$.

Proposition 3.3. Assume that (A2)-(A4) hold true and $\mu>0$. Let $F_{1}, F_{2}$ be two elements of $L^{1}(\Omega)$, and denote by $\theta_{i}$ the unique renormalized solution of $P\left(F_{i}\right)(i=1,2)$.

Then for any $K>0$

$$
\begin{aligned}
\mu \int_{\Omega}\left(\theta_{1}-\theta_{2}\right) T_{K}\left(\theta_{1}-\theta_{2}\right) \mathrm{d} x+\int_{\left\{\left|\theta_{1}-\theta_{2}\right|<K\right\}}\left(\mathbf{a}\left(x, D \theta_{1}\right)\right. & \left.-\mathbf{a}\left(x, D \theta_{2}\right)\right) \cdot\left(D \theta_{1}-D \theta_{2}\right) \mathrm{d} x \\
& \leq \int_{\Omega}\left(F_{1}-F_{2}\right) T_{K}\left(\theta_{1}-\theta_{2}\right) \mathrm{d} x
\end{aligned}
$$

Remark 3.4. Inequality (12) is obtained by plugging the admissible test function $h_{n}\left(\theta_{1}\right) h_{n}\left(\theta_{2}\right) T_{K}\left(\theta_{1}-\theta_{2}\right)$ in the difference of the equations $P\left(F_{1}\right)$ and $P\left(F_{2}\right)$ (that is licit in view of Proposition 3.1) where $h_{n}$ is a sequence of functions in $W^{1, \infty}(\mathbb{R})$ such that $h_{n}(r) \rightarrow 1$ as $n$ tends to $\infty$ and with compact support.

Due to Proposition 3.3 we deduce that

$$
\left\|\theta_{1}-\theta_{2}\right\|_{L^{1}(\Omega)} \leq \frac{1}{\mu}\left\|F_{1}-F_{2}\right\|_{L^{1}(\Omega)}
$$

and the continuity of the renormalized solution of $P(F)$ with respect to the datum $F$.

We recall the following lemma that can be proved by means of the estimates techniques of L. Boccardo and T. Gallouët [6] (see also [2]).

Lemma 3.5. Let $\theta$ be a measurable function defined on $\Omega$, that is finite almost everywhere in $\Omega$, and $M>0$ such that

$$
\begin{gathered}
\forall K>0, \quad T_{K}(\theta) \in H_{0}^{1}(\Omega) \quad \text { and } \\
\int_{\Omega}\left|D T_{K}(\theta)\right|^{2} \mathrm{~d} x \leq K M .
\end{gathered}
$$

Then $\theta \in W_{0}^{1, p}(\Omega)$ for any $1 \leq p<N /(N-1)$ and there exists a constant $C$ (depending on $\Omega$ and $p$ ) such that

$$
\|\theta\|_{W_{0}^{1, p}(\Omega)} \leq C M
$$

Gathering Proposition 3.1 and Lemma 3.5 we deduce the following corollary.

Corollary 3.6. Assume that (A2)-(A4) hold true and $\mu>0$. Let $F$ be an element of $L^{1}(\Omega)$ and $\theta$ the renormalized solution of $P(F)$. Then for any $1 \leq p<N /(N-1), \theta \in W_{0}^{1, p}(\Omega)$ and

$$
\|\theta\|_{W_{0}^{1, p}(\Omega)} \leq C\|F\|_{L^{1}(\Omega)}
$$

where $C$ is a constant only depending upon $\Omega, p$ and $\mathbf{a}$. 


\section{Existence of Small solutions of (1)-(3) For Small Data}

In this section we assume that the continuous function $f$ satisfies the following growth assumption

$$
\exists a \geq 0, \exists M>0, \quad \forall r \in \mathbb{R} \quad|f(r)| \leq a+M|r|^{\alpha}
$$

with $1 / 2<\alpha<N /(2(N-2))$ if $N \geq 3$ and $1 / 2<\alpha<+\infty$ if $N=2$.

Under this additional assumption, Theorem 4.1 insures the existence of at least a solution of Problem (1) -(3) for small data enough. Notice that on the one hand the upper bound of $\alpha$ in (14) is motivated in Introduction, on the other hand the lower bound permits us to exploit the small character on the data.

Theorem 4.1. Assume that (A1)-(A7) and (14) hold true. There exists a real positive number $\eta$ such that if $a+\|g\|_{L^{2}(\Omega)}<\eta$, then there exists at least a weak-renormalized solution of (11)-(3) such that

$$
\|u\|_{H_{0}^{1}(\Omega)}+\|\theta\|_{L^{2 \alpha}(\Omega)} \leq \omega(\eta)
$$

where $\omega(\eta)$ tends to zero as $\eta$ tends to zero.

Proof. The proof is divided into 2 steps. Step 1 is devoted to the construction of a fixed-point operator. In Step 2 we give a sufficient condition on the data in order to apply the Schauder fixed-point Theorem.

Step 1. Since the function $f$ is continuous and verifies growth assumption (14), under assumptions (A1), (A5) and (A7) the mapping

$$
\begin{aligned}
L^{2 \alpha}(\Omega) & \longmapsto H_{0}^{1}(\Omega) \\
\hat{\theta} & \longmapsto \hat{u}, \quad \text { where } \hat{u} \text { is the unique solution of the problem }
\end{aligned}
$$

$$
\left\{\begin{array}{c}
\lambda \hat{u}-\operatorname{div}(\mathbf{A}(x) D \hat{u}-f(\hat{\theta}))=g \quad \text { in } \Omega \\
\hat{u}=0 \quad \text { on } \partial \Omega,
\end{array}\right.
$$

is continuous and the coercivity of $\mathbf{A}$ implies that

$$
\int_{\Omega}|D \hat{u}|^{2} \mathrm{~d} x \leq C\left(\|g\|_{L^{2}(\Omega)}^{2}+\|f(\hat{\theta})\|_{\left(L^{2}(\Omega)^{N}\right)}^{2}\right)
$$

where $C$ is a generic constant independent of $\hat{\theta}$.

Let $\hat{\theta}$ be an element of $L^{2 \alpha}(\Omega)$ and $\hat{u}$ the unique element of $H_{0}^{1}(\Omega)$ solution of (15). Due to growth assumption (14) on $f$ and the regularity of $\hat{u}$, the field $(\mathbf{A}(x) D \hat{u}-f(\hat{\theta})) \cdot D \hat{u}$ belongs to $L^{1}(\Omega)$, and by Proposition 3.1 , let $\theta$ be the unique renormalized solution of the problem:

$$
\begin{array}{ll}
\mu \theta-\operatorname{div}(\mathbf{a}(x, D \theta))=(\mathbf{A}(x) D \hat{u}-f(\hat{\theta})) \cdot D \hat{u} & \text { in } \Omega \\
\theta=0 & \text { on } \partial \Omega .
\end{array}
$$

We denote by $\Gamma$ the mapping defined by $\theta=\Gamma(\hat{\theta})$.

Since $1<2 \alpha<N /(N-2)$ (and $1<2 \alpha<+\infty$ if $N=2$ ), let $q$ be a positive real number such that $2 \alpha<q^{*}<N /(N-2)$ (and $2 \alpha<q^{*}<+\infty$ if $N=2$ ), where $q^{*}$ denotes the Sobolev conjugate exponent $\left(1 / q^{*}=1 / q-1 / N\right)$.

Using the properties of the renormalized solutions (see Remark 3.4 and Corollary 3.6 ), the interpolation of $L^{2 \alpha}(\Omega)$ between $L^{1}(\Omega)$ and $L^{q^{*}}(\Omega)$ and the Rellich Kondrachov Theorem we 
deduce that $\Gamma$ is defined continuous and compact from $L^{2 \alpha}(\Omega)$ into itself. Moreover inequality (16)) and Corollary 3.6 imply that $\forall \hat{\theta} \in L^{2 \alpha}(\Omega)$, if $\theta=\Gamma(\hat{\theta})$ then

$$
\|\theta\|_{W_{0}^{1, q}(\Omega)} \leq C\left(\|g\|_{L^{2}(\Omega)}^{2}+\|f(\hat{\theta})\|_{\left(L^{2}(\Omega)\right)^{N}}^{2}\right)
$$

and growth assumption (14) on $f$ yields

$$
\|\theta\|_{W_{0}^{1, q}(\Omega)} \leq C\left(\|g\|_{L^{2}(\Omega)}^{2}+a^{2}+M^{2}\|\hat{\theta}\|_{L^{2 \alpha}(\Omega)}^{2 \alpha}\right)
$$

where $C$ is a constant independent of $\hat{\theta}$.

Step 2. Applying the Schauder fixed-point Theorem to the mapping $\Gamma$ reduces to show that there exists for instance a ball $B$ of $L^{2 \alpha}(\Omega)$ such that $\Gamma(B) \subset B$.

Let $\hat{\theta}$ be an element of $L^{2 \alpha}(\Omega)$ and $\theta=\Gamma(\hat{\theta})$. Since $1<2 \alpha<q^{*}<N /(N-2)$ (and $1<2 \alpha<q^{*}<+\infty$ if $N=2$ ), the Sobolev embedding Theorem and (19) lead to

$$
\|\theta\|_{L^{2 \alpha}(\Omega)} \leq C\left(\|g\|_{L^{2}(\Omega)}^{2}+a^{2}+M^{2}\|\hat{\theta}\|_{L^{2 \alpha}(\Omega)}^{2 \alpha}\right)
$$

where $C$ is a constant independent of $\hat{\theta}, g, a$ and $M$.

As $2 \alpha>1$, let $\eta>0$ and $R(\eta)>0$ such that

$$
\begin{gathered}
C\left(\eta+M^{2}(R(\eta))^{2 \alpha}\right)<R(\eta), \\
R(\eta)<2 C \eta .
\end{gathered}
$$

If

$$
\|g\|_{L^{2}(\Omega)}^{2}+a^{2}<\eta
$$

then we have

$$
\Gamma\left(B_{L^{2 \alpha}(\Omega)}(0, R(\eta))\right) \subset B_{L^{2 \alpha}(\Omega)}(0, R(\eta)) .
$$

Therefore, we may apply the Schauder fixed-point Theorem so that, there exists at least a solution $(u, \theta)$ of (11) (3) in the sense of Definition 2.2.

Moreover the choice of $R(\eta)$ and (16) imply that

$$
\|u\|_{H_{0}^{1}(\Omega)}+\|\theta\|_{L^{2 \alpha}(\Omega)} \leq \omega(\eta)
$$

where $\omega(\eta)$ tends to zero as $\eta$ tends to zero.

The proof of Theorem 4.1 is complete.

\section{Existence of a solution of (1)-(3) FOR more general data}

In order to remove the small character on the data of the previous section, we suppose by now more restrictive hypotheses on the behavior of $f$, which are on $\mathbb{R}^{+}$

$$
\left\{\begin{array}{l}
\lim _{r \rightarrow+\infty} \frac{|f(r)|}{r^{(N+2) /(2 N)}}=0 \quad \text { if } N \geq 3, \\
\forall r \in \mathbb{R}^{+} \quad|f(r)| \leq a+M|r| \quad \text { if } N=2 \text { with } a \geq 0 \text { and } M \geq 0,
\end{array}\right.
$$

that is more restrictive than (14) since $(N+2) /(2 N)<N /(2(N-2)))$, and on $\mathbb{R}^{-}$a behavior of which the model case is $f=0$ for $r<r_{0} \leq 0$. (see hypotheses (23) and (24) below). 
Theorem 5.1. Assume that assumptions (A1)-(A7) and (22) hold true. Moreover assume that the continuous function $f$ is such that

$$
\lim _{r \rightarrow-\infty} \frac{|f(r)|}{\sqrt{|r|}}=0
$$

Then there exists at least a weak-renormalized solution of (1)-(3).

In the case where the function $f$ has a zero on $\mathbb{R}^{-}$, the structure of equation (2) allows us to remove the growth assumption on $f$ on $\mathbb{R}^{-}$and give an additional property on $\theta$.

Theorem 5.2. Assume that assumptions (A1)-(A7) and (22) hold true. Moreover assume that the continuous function $f$ satisfies:

$$
\exists r_{0} \in \mathbb{R}^{-} \text {such that } f\left(r_{0}\right)=0 .
$$

Then there exists at least a weak-renormalized solution $(u, \theta)$ of (1)-(3) such that $\theta \geq r_{0}$ almost everywhere in $\Omega$.

Remark 5.3. The existence results of Theorems 5.1 and 5.2 were announced in [16] (see also [15]) under more restrictive hypotheses on the function $f$. Let us notice that when $N=2$ linear growth on $\mathbb{R}^{+}$is allowed for $f$.

Before to prove Theorem 5.1, we give a technical lemma.

Lemma 5.4. Assume that (22) holds true. Let $\theta$ be a measurable function defined on $\Omega$ such that

$$
\begin{gathered}
\theta \in L^{1}(\Omega), \\
\forall K>0 \quad T_{K}(\theta) \in H_{0}^{1}(\Omega), \\
\exists C_{1}>0 \text { such that } \forall K>0 \quad \frac{1}{K} \int_{\Omega}\left|D T_{K}(\theta)\right|^{2} \mathrm{~d} x<C_{1}\left(\int_{\{0 \leq \theta \leq K\}} f^{2}(\theta) \mathrm{d} x+1\right) .
\end{gathered}
$$

Then for any $1 \leq q<N /(N-2)$ (and $1 \leq q<+\infty$ if $N=2)$, there exists a constant $C^{\prime}$, only depending upon $q, \Omega,\|\theta\|_{L^{1}(\Omega)}, C_{1}$ and $f$ such that

$$
\|\theta\|_{L^{q}(\Omega)} \leq C^{\prime} .
$$

Sketch of the proof. The proof relies on estimate techniques of L. Boccardo and T. Gallouët [6] (see also [2]). If $N=2$ we use the limit case of the Sobolev embedding Theorem (see [1], [14] for instance) that allows us to reach linear growth on $\mathbb{R}^{+}$for the function $f$.

Case $N \geq 3$. Let $n$ be an element of $\mathbb{N}$, that will fixed in the sequel, and let $q$ be such that $1<q<N /(N-2)$. Hypothesis (22) gives that

$$
\forall r \in\left[2^{n},+\infty\left[|f(r)| \leq \omega(n) r^{(N+2) / 2 N},\right.\right.
$$

where $\omega(n)$ tends to zero as $n$ tends to infinity.

As $\theta$ is finite almost everywhere in $\Omega$, we have

$$
\begin{aligned}
\int_{\Omega}|\theta|^{q} \mathrm{~d} x & \leq 2^{n q}|\Omega|+\sum_{k=n}^{+\infty} \int_{\left\{2^{k}<|\theta| \leq 2^{k+1}\right\}}|\theta|^{q} \mathrm{~d} x \\
& \leq 2^{n q}|\Omega|+\sum_{k=n}^{+\infty}\left(\frac{1}{2^{k}}\right)^{2^{*}-q} \int_{\Omega}\left|T_{2^{k+1}}(\theta)\right|^{2^{*}} \mathrm{~d} x,
\end{aligned}
$$


where $2^{*}$ denotes the Sobolev conjugate exponent $\left(1 / 2^{*}=1 / 2-1 / N\right)$.

The Sobolev embedding Theorem and (26) (with $K=2^{k+1}$ ) yield

$$
\int_{\Omega}|\theta|^{q} \mathrm{~d} x \leq 2^{n q}|\Omega|+C C_{1}^{2^{*} / 2} \sum_{k=n}^{+\infty}\left(\frac{1}{2^{k}}\right)^{2^{*}-q}\left(2^{k+1} \int_{\left\{0 \leq \theta \leq 2^{k+1}\right\}} f^{2}(\theta) \mathrm{d} x+2^{k+1}\right)^{2^{*} / 2},
$$

where $C$ is a constant depending on $\Omega$.

Using (28) we obtain

$$
\begin{aligned}
\int_{\Omega}|\theta|^{q} \mathrm{~d} x \leq 2^{n q}|\Omega|+C\left(2 C_{1}\right)^{2^{*} / 2} \sum_{k=n}^{+\infty}\left(\frac{1}{2^{k}}\right)^{2^{*} / 2-q} & \left(|\Omega| \max _{r \in\left[0,2^{n}\right]}|f(r)|^{2}+1\right. \\
& \left.+\omega(n) \int_{\left\{2^{n} \leq \theta \leq 2^{k+1}\right\}}|\theta|^{(N+2) / N} \mathrm{~d} x\right)^{2^{*} / 2} .
\end{aligned}
$$

On the one hand Hölders inequality gives, $\forall n \leq k<+\infty$,

$$
\int_{\left\{2^{n} \leq \theta \leq 2^{k+1}\right\}}|\theta|^{(N+2) / N} \mathrm{~d} x \leq\left(\|\theta\|_{L^{1}(\Omega)}\right)^{2 / N}\left(\int_{\left\{2^{n} \leq \theta \leq 2^{k+1}\right\}}|\theta|^{N /(N-2)} \mathrm{d} x\right)^{2 / 2^{*}},
$$

on the other hand as $q<N /(N-2)=2^{*} / 2$, the series $\sum_{k=n}^{+\infty}\left(\frac{1}{2^{k}}\right)^{2^{*} / 2-q}$ is convergent and we have

$$
\sum_{k=n}^{+\infty}\left(\frac{1}{2^{k}}\right)^{2^{*} / 2-q} \int_{\left\{2^{n} \leq \theta \leq 2^{k+1}\right\}}|\theta|^{N /(N-2)} \mathrm{d} x \leq C(q) \int_{\Omega}|\theta|^{q} \mathrm{~d} x,
$$

where $C(q)$ is a constant only depending on $q$.

After a few computations, from inequality (29) together with (30) and (31) it follows that

$$
\int_{\Omega}|\theta|^{q} \mathrm{~d} x \leq M_{1}\left(n, q, \Omega, f, C_{1}\right)+(\omega(n))^{2^{*} / 2} M_{2}\left(\|\theta\|_{L^{1}(\Omega)}, q, C_{1}, \Omega\right) \int_{\Omega}|\theta|^{q} \mathrm{~d} x,
$$

where $M_{1}$ is a constant only depending on $n, q, \Omega, f$ and $C_{1}$, and $M_{2}$ is a constant only depending on $\|\theta\|_{L^{1}(\Omega)}, q, C_{1}$ and $\Omega$.

Therefore since $\omega(n)$ tends to zero as $n$ tends to infinity, we can choose $n$ such that $(\omega(n))^{2^{*} / 2} M_{2}\left(\|\theta\|_{L^{1}(\Omega)}, q, C_{1}, \Omega\right)<1 / 2$ and then (32) yields

$$
\int_{\Omega}|\theta|^{q} \mathrm{~d} x \leq 2 M_{1}\left(n, q, \Omega, f, C_{1}\right)
$$

that is (27).

Case $N=2$. Let $a$ and $M$ be two non negative real numbers such that

$$
\forall r \in \mathbb{R}^{+} \quad|f(r)| \leq a+M|r| .
$$

Using similar techniques as in the previous case, we obtain

$$
\begin{aligned}
\int_{\Omega}\left|\frac{D \theta}{1+|\theta|}\right|^{2} \mathrm{~d} x & \leq C\left(1+\sum_{k=0}^{+\infty} \frac{1}{2^{k+1}} \int_{\left\{0<\theta \leq 2^{k+1}\right\}}|\theta|^{2} \mathrm{~d} x\right) \\
& \leq C\left(1+\int_{\Omega}|\theta| \mathrm{d} x\right)
\end{aligned}
$$


where $C$ is a constant only depending on $\Omega, a, M$ and $C_{1}$.

It follows that

$$
\|\ln (1+|\theta|)\|_{H_{0}^{1}(\Omega)} \leq C
$$

where $C$ is a constant only depending on $\Omega, a, M, C_{1}$ and $\|\theta\|_{L^{1}(\Omega)}$.

Making use of Theorem 7.15 from [14], let $C_{2}$ and $C_{3}$ be two positive real numbers only depending on $N$, such that

$$
\int_{\Omega} \exp \left[\left(\frac{\ln (1+|\theta|)}{C_{2}\|D(\ln (1+|\theta|))\|_{L^{2}(\Omega)}}\right)^{2}\right] \mathrm{d} x \leq C_{3}|\Omega|
$$

Therefore from (34) we have

$$
\int_{\Omega} \exp \left[\left(\frac{\ln (1+|\theta|)}{C_{2} C}\right)^{2}\right] \mathrm{d} x \leq C_{3}|\Omega|,
$$

where $C, C_{2}$ and $C_{3}$ does not depend on $\theta$.

If follows that for any $1 \leq q<+\infty$ there exists $C^{\prime}>0$ only depending on $f, \Omega, C_{1}, C_{2}$, $C_{3},\|\theta\|_{L^{1}(\Omega)}$ and $q$ such that

$$
\|\theta\|_{L^{q}(\Omega)} \leq C^{\prime}
$$

The proof of Lemma 5.4 is complete.

We are now in a position to prove Theorem [5.1.

Proof of Theorem [5.1. The proof is divided into 3 steps. In Step 1 we consider a solution $\left(u^{\varepsilon}, \theta^{\varepsilon}\right)$ of the approximate problem (11)-(3) with $f^{\varepsilon}=f \circ T_{1 / \varepsilon}(\varepsilon>0)$ in place of $f$ and we derive a few preliminary estimates. In Step 2, using the coupling between the unknowns $u^{\varepsilon}$ and $\theta^{\varepsilon}$, we establish an important equality that first implies an $L^{1}(\Omega)$-estimate on $\theta^{\varepsilon}$. In Step 3, we make use of Lemma 5.4 to obtain an $L^{2}(\Omega)$-estimate on $f^{\varepsilon}\left(\theta^{\varepsilon}\right)$ and, at last, we pass to the limit in the approximate problem.

Step 1. For $\varepsilon>0$, we consider the following system

$$
\begin{array}{ll}
\lambda u^{\varepsilon}-\operatorname{div}\left(\mathbf{A}(x) D u^{\varepsilon}-f^{\varepsilon}\left(\theta^{\varepsilon}\right)\right)=g & \text { in } \Omega, \\
\mu \theta^{\varepsilon}-\operatorname{div}\left(\mathbf{a}\left(x, D \theta^{\varepsilon}\right)\right)=\left(\mathbf{A}(x) D u^{\varepsilon}-f^{\varepsilon}\left(\theta^{\varepsilon}\right)\right) \cdot D u^{\varepsilon} & \text { in } \Omega, \\
u^{\varepsilon}=0 \quad \theta^{\varepsilon}=0 & \text { on } \partial \Omega .
\end{array}
$$

Analyzing the proof of Theorem 4.1 allows us to show that there exists at least a weakrenormalized solution $\left(u^{\varepsilon}, \theta^{\varepsilon}\right)$ of (35)-(37). Indeed as the continuous function $f^{\varepsilon}$ is bounded, the mapping $\Gamma$ constructed in the proof of Theorem 4.1 is continuous and compact from $L^{1}(\Omega)$ into a bounded subset of $L^{1}(\Omega)$. Then the Schauder fixed-point Theorem allows us to conclude.

For $\varepsilon>0$, let $\left(u^{\varepsilon}, \theta^{\varepsilon}\right)$ be a weak-renormalized solution of (35)-(37). It follows from (16) that

$$
\begin{gathered}
\int_{\Omega}\left|D u^{\varepsilon}\right|^{2} \mathrm{~d} x<C\left(1+\left\|f^{\varepsilon}\left(\theta^{\varepsilon}\right)\right\|_{\left(L^{2}(\Omega)\right)^{N}}^{2}\right), \\
\left\|\left(\mathbf{A}(x) D u^{\varepsilon}-f^{\varepsilon}\left(\theta^{\varepsilon}\right)\right) \cdot D u^{\varepsilon}\right\|_{L^{1}(\Omega)} \leq C\left(1+\left\|f^{\varepsilon}\left(\theta^{\varepsilon}\right)\right\|_{\left(L^{2}(\Omega)\right)^{N}}^{2}\right),
\end{gathered}
$$


and, recalling that $\theta^{\varepsilon}$ is a renormalized solution of (36)-(37) and using Proposition 3.1 ,

$$
\int_{\Omega}\left|D T_{K}\left(\theta^{\varepsilon}\right)\right|^{2} \mathrm{~d} x \leq C K\left(1+\left\|f^{\varepsilon}\left(\theta^{\varepsilon}\right)\right\|_{\left(L^{2}(\Omega)\right)^{N}}^{2}\right) \quad \forall K>0,
$$

where $C$ is a constant independent of $\varepsilon$ and $K$.

Remark 5.5. From Corollary 3.6 we obtain that for any $1 \leq p<N /(N-1)$

$$
\left\|\theta^{\varepsilon}\right\|_{W_{0}^{1, p}(\Omega)} \leq C\left(1+\left\|f^{\varepsilon}\left(\theta^{\varepsilon}\right)\right\|_{\left(L^{2}(\Omega)\right)^{N}}^{2}\right) .
$$

In the case where $\lim _{r \rightarrow+\infty}|f(r)|^{2} / r=0$, deriving an $L^{q}$-estimate for any $1 \leq q<N /(N-2)$ (and $1 \leq q<+\infty$ if $N=2$ ) is an easy task. But under hypothesis (22), a new estimate on $\theta^{\varepsilon}$ is necessary to obtain a upper bound on $\left\|\theta^{\varepsilon}\right\|_{L^{1}(\Omega)}$ and more generally on $\left\|\theta^{\varepsilon}\right\|_{L^{q}(\Omega)}$.

Step 2. For $K>0$, since $T_{K}\left(\theta^{\varepsilon}\right) \in L^{\infty}(\Omega) \cap H_{0}^{1}(\Omega)$ and $D T_{K}\left(\theta^{\varepsilon}\right)=0$ almost everywhere on $\left\{x:\left|\theta^{\varepsilon}(x)\right| \geq K\right\}$ Proposition 3.1 (with $w=T_{K}\left(\theta^{\varepsilon}\right) / K$ ) gives that

$\mu \int_{\Omega} \frac{T_{K}\left(\theta^{\varepsilon}\right)}{K} \theta^{\varepsilon} \mathrm{d} x+\frac{1}{K} \int_{\Omega} \mathbf{a}\left(x, D \theta^{\varepsilon}\right) \cdot D T_{K}\left(\theta^{\varepsilon}\right) \mathrm{d} x=\int_{\Omega} \frac{T_{K}\left(\theta^{\varepsilon}\right)}{K}\left(\mathbf{A}(x) D u^{\varepsilon}-f^{\varepsilon}\left(\theta^{\varepsilon}\right)\right) \cdot D u^{\varepsilon} \mathrm{d} x$.

Plugging the test function $u^{\varepsilon}$ in (35) and summing the result to the previous equality yield

$$
\begin{aligned}
& \lambda \int_{\Omega}\left(u^{\varepsilon}\right)^{2} \mathrm{~d} x+\mu \int_{\Omega} \frac{T_{K}\left(\theta^{\varepsilon}\right)}{K} \theta^{\varepsilon} \mathrm{d} x+\frac{1}{K} \int_{\Omega} \mathbf{a}\left(x, D \theta^{\varepsilon}\right) \cdot D T_{K}\left(\theta^{\varepsilon}\right) \mathrm{d} x \\
& \quad+\int_{\Omega} \frac{K-T_{K}\left(\theta^{\varepsilon}\right)}{K} \mathbf{A}(x) D u^{\varepsilon} \cdot D u^{\varepsilon} \mathrm{d} x=\int_{\Omega} \frac{K-T_{K}\left(\theta^{\varepsilon}\right)}{K} f^{\varepsilon}\left(\theta^{\varepsilon}\right) \cdot D u^{\varepsilon} \mathrm{d} x+\int_{\Omega} g u^{\varepsilon} \mathrm{d} x .
\end{aligned}
$$

Since $\lambda>0$ and $K-T_{K}\left(\theta^{\varepsilon}\right)$ is a non negative function, the coercivity of $\mathbf{a}$ and $\mathbf{A}$ together with Young's inequality lead to

$$
\begin{array}{rl}
\int_{\Omega}\left(u^{\varepsilon}\right)^{2} \mathrm{~d} & x+\int_{\Omega} \frac{T_{K}\left(\theta^{\varepsilon}\right)}{K} \theta^{\varepsilon} \mathrm{d} x+\frac{1}{K} \int_{\Omega}\left|D T_{K}\left(\theta^{\varepsilon}\right)\right|^{2} \mathrm{~d} x \\
& +\int_{\Omega} \frac{K-T_{K}\left(\theta^{\varepsilon}\right)}{K}\left|D u^{\varepsilon}\right|^{2} \mathrm{~d} x \leq C\left(\int_{\Omega} \frac{K-T_{K}\left(\theta^{\varepsilon}\right)}{K}\left|f^{\varepsilon}\left(\theta^{\varepsilon}\right)\right|^{2} \mathrm{~d} x+\int_{\Omega} g^{2} \mathrm{~d} x\right),
\end{array}
$$

where $C$ is a constant independent of $\varepsilon$ and $K$.

As $\forall \varepsilon>0$

$$
\begin{gathered}
\quad \theta^{\varepsilon} \frac{T_{K}\left(\theta^{\varepsilon}\right)}{K} \stackrel{K \rightarrow 0}{\longrightarrow}\left|\theta^{\varepsilon}\right| \quad \text { almost everywhere in } \Omega \text { and } \\
\frac{K-T_{K}\left(\theta^{\varepsilon}\right)}{K} \stackrel{K \rightarrow 0}{\longrightarrow} 2 \mathbb{1}_{\left\{\theta^{\varepsilon}<0\right\}}+\mathbb{1}_{\left\{\theta^{\varepsilon}=0\right\}} \quad \text { almost everywhere in } \Omega,
\end{gathered}
$$

passing to the limit as $K$ tends to zero in inequality (42) gives that, $\forall \varepsilon>0$,

$$
\int_{\Omega}\left(u^{\varepsilon}\right)^{2} \mathrm{~d} x+\int_{\Omega}\left|\theta^{\varepsilon}\right| \mathrm{d} x \leq C\left(\int_{\Omega}\left|f^{\varepsilon}\left(\theta^{\varepsilon}\right)\right|^{2} \mathbb{1}_{\left\{\theta^{\varepsilon} \leq 0\right\}} \mathrm{d} x+\int_{\Omega} g^{2} \mathrm{~d} x\right)
$$

where $C$ is constant independent of $\varepsilon$.

Due to assumption (23) on the behavior of $f$ on $\mathbb{R}^{-}, \forall \eta>0, \exists C_{\eta}>0$ such that, $\forall r \in \mathbb{R}^{-}$, $|f(r)|^{2} \leq \eta|r|+C_{\eta}$. If we choose $\eta$ sufficiently small, then inequality (43) implies that there exists $C_{1}>0$ such that, $\forall \varepsilon>0$,

$$
\int_{\Omega}\left(u^{\varepsilon}\right)^{2} \mathrm{~d} x+\int_{\Omega}\left|\theta^{\varepsilon}\right| \mathrm{d} x \leq C_{1} .
$$


Remark 5.6. Inequality (43) shows that $\theta^{\varepsilon}$ and $u^{\varepsilon}$ are controlled respectively in $L^{1}(\Omega)$ and in $L^{2}(\Omega)$ if $f^{\varepsilon}\left(\theta^{\varepsilon}\right)$ is controlled in $L^{2}(\Omega)$ only on the subset of $\Omega$ where $\theta^{\varepsilon} \leq 0$. Inequality (43) is also used to prove uniqueness result (see Theorem 6.1).

Step 3. As all the terms in the left hand side of (42) are non negative, one has, $\forall \varepsilon>0$ and $\forall K>0$,

$$
\begin{aligned}
\frac{1}{K} \int_{\Omega}\left|D T_{K}\left(\theta^{\varepsilon}\right)\right|^{2} \mathrm{~d} x & \leq C\left(\int_{\Omega} \frac{K-T_{K}\left(\theta^{\varepsilon}\right)}{K}\left|f^{\varepsilon}\left(\theta^{\varepsilon}\right)\right|^{2} \mathrm{~d} x+\int_{\Omega} g^{2} \mathrm{~d} x\right) \\
& \leq C\left(\int_{\left\{\theta^{\varepsilon}<0\right\}} 2\left|f^{\varepsilon}\left(\theta^{\varepsilon}\right)\right|^{2} \mathrm{~d} x+\int_{\left\{0 \leq \theta^{\varepsilon}<K\right\}}\left|f^{\varepsilon}\left(\theta^{\varepsilon}\right)\right|^{2} \mathrm{~d} x+\int_{\Omega} g^{2} \mathrm{~d} x\right),
\end{aligned}
$$

since $K-T_{K}\left(\theta^{\varepsilon}\right)=0$ almost everywhere on $\left\{x: \theta^{\varepsilon}(x) \geq K\right\}$.

Therefore growth assumption (22) on $f$ and estimate (44) imply that there exists $C_{2}>0$ such that $\forall \varepsilon>0, \forall K>0$

$$
\frac{1}{K} \int_{\Omega}\left|D T_{K}\left(\theta^{\varepsilon}\right)\right|^{2} \mathrm{~d} x \leq C_{2}\left(\int_{\left\{0 \leq \theta^{\varepsilon} \leq K\right\}}\left|f^{\varepsilon}\left(\theta^{\varepsilon}\right)\right|^{2} \mathrm{~d} x+1\right) .
$$

Let us denote $f^{*}$ the real-valued function defined by $f^{*}(r)=\sup _{0 \leq r^{\prime} \leq r}\left|f\left(r^{\prime}\right)\right|, \forall r \in \mathbb{R}^{+}$. The function $f^{*}$ satisfies (22) and $\forall \varepsilon>0, \forall K>0$

$$
\frac{1}{K} \int_{\Omega}\left|D T_{K}\left(\theta^{\varepsilon}\right)\right|^{2} \mathrm{~d} x \leq C_{2}\left(\int_{\left\{0 \leq \theta^{\varepsilon}<K\right\}}\left|f^{*}\left(\theta^{\varepsilon}\right)\right|^{2} \mathrm{~d} x+1\right) .
$$

Since $C_{1}$ and $C_{2}$ are independent of $\varepsilon$ and $K$, from (43) and the above inequality we can apply Lemma 5.4 to $\theta^{\varepsilon}, \forall \varepsilon>0$. It follows that the sequence $\theta^{\varepsilon}$ is bounded in $L^{q}(\Omega)$ for any $1 \leq q<N /(N-2)$ (and $1 \leq q<+\infty$ if $N=2$ ). In particular, growth assumptions (22) and (23) on $f$ imply that

$$
f^{\varepsilon}\left(\theta^{\varepsilon}\right) \text { is bounded in }\left(L^{2}(\Omega)\right)^{N},
$$

and from (41) we obtain that for any $1 \leq p<N /(N-1)$

$$
\theta^{\varepsilon} \text { is bounded in } W_{0}^{1, p}(\Omega) \text {. }
$$

By the Rellich Kondrachov Theorem, let $\theta$ be a measurable function defined from $\Omega$ into $\mathbb{R}$ such that, up to a subsequence, $\forall 1 \leq q<N /(N-2)$ (and $1<q<+\infty$ if $N=2$ )

$$
\theta^{\varepsilon} \longrightarrow \theta \text { in } L^{q}(\Omega) \text { and almost everywhere in } \Omega \text {, as } \varepsilon \text { tends to zero. }
$$

Since $(N+2) / N<N /(N-2)$, the continuity of $f$, growth assumptions (22) and (23) and (46) allow us to deduce, by a standard equiintegrability argument, that

$$
f^{\varepsilon}\left(\theta^{\varepsilon}\right) \longrightarrow f(\theta) \text { in }\left(L^{2}(\Omega)\right)^{N} \text { as } \varepsilon \text { tends to zero. }
$$

Next, using the linear character of equation (35) with respect to $u^{\varepsilon}$ together with (47) it is easy to show that

$$
u^{\varepsilon} \longrightarrow u \text { in } H_{0}^{1}(\Omega) \text { as } \varepsilon \text { tends to zero, }
$$

and then, $(u, \theta)$ satisfies equation (1) in $\mathcal{D}^{\prime}(\Omega)$ with $u \in H_{0}^{1}(\Omega)$ and $f(\theta) \in\left(L^{2}(\Omega)\right)^{N}$.

It follows that

$$
\left(\mathbf{A}(x) D u^{\varepsilon}-f^{\varepsilon}\left(\theta^{\varepsilon}\right)\right) \cdot D u^{\varepsilon} \longrightarrow(\mathbf{A}(x) D u-f(\theta)) \cdot D u \quad \text { in } L^{1}(\Omega) \text { as } \varepsilon \text { tends to zero. }
$$


As far as equation (36) is concerned, the continuity of renormalized solution with respect to the data implies that $\theta$ is a renormalized solution of (2).

The proof of Theorem 5.1 is complete.

We now prove Theorem 5.2 .

Proof of Theorem 5.2. Let $\widetilde{f}$ be the function defined by

$$
\tilde{f}(r)=\left\{\begin{aligned}
0 & \text { if } r \leq r_{0} \\
f(r) & \text { if } r>r_{0}
\end{aligned}\right.
$$

The function $\tilde{f}$ is continuous and satisfies assumptions (22) and (23). Making use of Theorem 5.1, let $(u, \theta)$ be a weak-renormalized solution of system (1)-(3) with $\tilde{f}$ in place of $f$.

Our aim now is to prove that

$$
\theta \geq r_{0} \quad \text { almost everywhere in } \Omega .
$$

For $K>0$, let $H$ be the function defined by $H(r)=-T_{K}^{-}\left(r-r_{0}\right), \forall r \in \mathbb{R}$. We have $H \in W^{1, \infty}(\mathbb{R}), H^{\prime}(r)=\mathbb{1}_{\left\{-K+r_{0}<r<r_{0}\right\}}$, so $H^{\prime}$ has a compact support. Since $r_{0} \leq 0$, it follows that $H(\theta) \in L^{\infty}(\Omega) \cap H_{0}^{1}(\Omega)$ and recalling that $\theta$ is a renormalized solution of (2), Proposition 3.1 with $w=H(\theta)$ leads to

$$
\mu \int_{\Omega} \theta H(\theta) \mathrm{d} x+\int_{\left\{-K+r_{0}<\theta<r_{0}\right\}} \mathbf{a}(x, D \theta) \cdot D \theta \mathrm{d} x=-\int_{\Omega}(\mathbf{A}(x) D u-\widetilde{f}(\theta)) \cdot D u H(\theta) \mathrm{d} x .
$$

The definitions of $H$ and $\widetilde{f}$ imply that $\widetilde{f}(r) H(r)=0, \forall r \in \mathbb{R}$, and because $H(r) \leq 0$ the coercivity of $\mathbf{a}$ and $\mathbf{A}$ gives

$$
\int_{\Omega}|\theta| T_{K}^{-}\left(\theta-r_{0}\right) \mathrm{d} x \leq 0
$$

It follows that

$$
\theta \geq r_{0} \quad \text { almost everywhere in } \Omega,
$$

and according to the definition of $\widetilde{f}$,

$$
\widetilde{f}(\theta)=f(\theta) \text { almost everywhere in } \Omega .
$$

Hence $(u, \theta)$ is a weak-renormalized solution of (11)-(3).

\section{UNIQUENESS RESULTS}

In this section we assume that

$$
f(0)=0,
$$

and we give the following uniqueness result of a small solution $(u, \theta)$ of (1) $-(3)$ such that $\theta \geq 0$ almost everywhere in $\Omega$ under additional assumptions on $f$, a and $N$. 
Theorem 6.1. Assume that assumptions (A1)-(A7), (22) and (48) hold true. Moreover assume that

$$
\begin{gathered}
N=2 \quad \text { or } \quad N=3, \\
\left(\mathbf{a}(x, \xi)-\mathbf{a}\left(x, \xi^{\prime}\right)\right) \cdot\left(\xi-\xi^{\prime}\right) \geq \delta\left|\xi-\xi^{\prime}\right|^{2} \quad \text { almost everywhere in } \Omega, \forall \xi, \xi^{\prime} \in \mathbb{R}^{N}, \\
\exists L>0 \text { such that } \forall r, r^{\prime} \in \mathbb{R}^{+} \quad\left|f(r)-f\left(r^{\prime}\right)\right| \leq L\left|r-r^{\prime}\right| .
\end{gathered}
$$

There exists $\eta>0$ such that if $\|g\|_{L^{2}(\Omega)}<\eta$, then the weak-renormalized solution $(u, \theta)$ of (1) -(3), such that $\theta \geq 0$ almost everywhere in $\Omega$, is unique.

Proof of Theorem 6.1. From Theorem 5.2 let $\left(u_{1}, \theta_{1}\right)$ and $\left(u_{2}, \theta_{2}\right)$ be two weak-renormalized solutions of (11)-(3) such that $\theta_{1} \geq 0$ and $\theta_{2} \geq 0$ almost everywhere in $\Omega$.

The aim is to prove that

$$
\left\|\theta_{1}-\theta_{2}\right\|_{L^{2}(\Omega)} \leq \omega\left(\|g\|_{L^{2}(\Omega)}\right)\left\|\theta_{1}-\theta_{2}\right\|_{L^{2}(\Omega)},
$$

where $\omega$ is independent of $\theta_{1}$ and $\theta_{2}$ and is such that $\omega(r)$ tends to zero as $r$ tends to zero.

We denote by $F_{i}$ the term $\left(\mathbf{A}(x) D u_{i}-f\left(\theta_{i}\right)\right) \cdot D u_{i}$, for $i=1,2$. Proposition 3.3 and (50) give

$$
\forall K>0, \quad \delta \int_{\left\{\left|\theta_{1}-\theta_{2}\right|<K\right\}}\left|D \theta_{1}-D \theta_{2}\right|^{2} \mathrm{~d} x \leq K \int_{\Omega}\left|F_{1}-F_{2}\right| \mathrm{d} x .
$$

From a result of [9], it follows that $T_{K}\left(\theta_{1}-\theta_{2}\right) \in H_{0}^{1}(\Omega)$ for any $K>0$. As $N=2$ or $N=3$ there exists $1<p<N /(N-1)$ such that $p^{*}=2$ and so Lemma 3.5 and the above inequality imply that

$$
\left\|\theta_{1}-\theta_{2}\right\|_{L^{2}(\Omega)} \leq C\left\|F_{1}-F_{2}\right\|_{L^{1}(\Omega)},
$$

where $C$ is a generic constant independent of $i$ and $g$.

A calculus leads to

$$
\begin{aligned}
\left\|F_{1}-F_{2}\right\|_{L^{1}(\Omega)} \leq & \|A\|_{\left(L^{\infty}(\Omega)\right)^{N \times N}}\left\|D u_{1}-D u_{2}\right\|_{\left(L^{2}(\Omega)\right)^{N}} \times\left\|D u_{1}+D u_{2}\right\|_{\left(L^{2}(\Omega)\right)^{N}} \\
& +\left\|f\left(\theta_{1}\right)-f\left(\theta_{2}\right)\right\|_{\left(L^{2}(\Omega)\right)^{N}} \times\left\|D u_{1}\right\|_{\left(L^{2}(\Omega)\right)^{N}} \\
& +\left\|f\left(\theta_{1}\right)\right\|_{\left(L^{2}(\Omega)\right)^{N}} \times\left\|D u_{1}-D u_{2}\right\|_{\left(L^{2}(\Omega)\right)^{N}} .
\end{aligned}
$$

The linear character of equation (11) gives

$$
\begin{gathered}
\left\|D u_{1}-D u_{2}\right\|_{\left(L^{2}(\Omega)\right)^{N}} \leq C\left\|f\left(\theta_{1}\right)-f\left(\theta_{2}\right)\right\|_{\left(L^{2}(\Omega)\right)^{N}}, \\
\left\|D u_{i}\right\|_{\left(L^{2}(\Omega)\right)^{N}} \leq C\left(\left\|f\left(\theta_{i}\right)\right\|_{\left(L^{2}(\Omega)\right)^{N}}+\|g\|_{L^{2}(\Omega)}\right), \quad \text { for } i=1,2 .
\end{gathered}
$$

Using (51) and the above inequalities we obtain

$$
\left\|F_{1}-F_{2}\right\|_{L^{1}(\Omega)} \leq C\left(\left\|f\left(\theta_{1}\right)\right\|_{\left(L^{2}(\Omega)\right)^{N}}+\left\|f\left(\theta_{2}\right)\right\|_{\left(L^{2}(\Omega)\right)^{N}}+\|g\|_{L^{2}(\Omega)}\right)\left\|\theta_{1}-\theta_{2}\right\|_{L^{2}(\Omega)},
$$

and therefore (52) reduces to prove that

$$
\left\|f\left(\theta_{i}\right)\right\|_{\left(L^{2}(\Omega)\right)^{N}} \leq \omega\left(\|g\|_{L^{2}(\Omega)}\right), \quad \text { for } i=1,2,
$$

where $\omega$ is independent of $i$ and is such that $\omega(r)$ tends to zero as $r$ tends to zero.

Since $\theta_{i} \geq 0$ almost everywhere in $\Omega$, (43) implies that

$$
\left\|\theta_{i}\right\|_{L^{1}(\Omega)} \leq C\|g\|_{L^{2}(\Omega)} \text { for } i=1,2,
$$


and from (42) we obtain, for $i=1,2$,

$$
\forall K>0 \quad \frac{1}{K} \int_{\Omega}\left|D T_{K}\left(\theta_{i}\right)\right|^{2} \mathrm{~d} x \leq C\left(\int_{\left\{0 \leq \theta_{i} \leq K\right\}}\left|f\left(\theta_{i}\right)\right|^{2} \mathrm{~d} x+\|g\|_{L^{2}(\Omega)}^{2}\right) .
$$

So if $\|g\|_{L^{2}(\Omega)} \leq 1$, (56) and (57) together with Lemma 5.4 allow us to deduce that, $\forall 1 \leq q<$ $N /(N-2)$ (and $q<+\infty$ if $N=2$ ),

$$
\left\|\theta_{i}\right\|_{L^{q}(\Omega)} \leq C(q)
$$

where $C(q)$ is a constant independent of $i$ and $g$.

Using (22), (48) and (51), let $M>0$ such that

$$
\forall r \in \mathbb{R}^{+} \quad|f(r)| \leq M r^{(N+2) /(2 N)} .
$$

By interpolation between $L^{1}(\Omega)$ and $L^{(N+1) /(N-1)}(\Omega)$ we have, for $i=1,2$,

$$
\left\|f\left(\theta_{i}\right)\right\|_{\left(L^{2}(\Omega)\right)^{N}} \leq M\left(\left\|\theta_{i}\right\|_{L^{\frac{N+2}{N}}(\Omega)}\right)^{\frac{N}{2(N+2)}} \leq M\left(\left\|\theta_{i}\right\|_{L^{1}(\Omega)}\right)^{\frac{1}{2 N}}\left(\left\|\theta_{i}\right\|_{L^{\frac{N+1}{N-1}}(\Omega)}\right)^{\frac{N+1}{2 N}},
$$

and using (56) and (58) (indeed $\left.\frac{N+1}{N-1}<\frac{N}{N-2}\right)$, if $\|g\|_{L^{2}(\Omega)} \leq 1$ then we have

$$
\left\|f\left(\theta_{i}\right)\right\|_{\left(L^{2}(\Omega)\right)^{N}} \leq C\|g\|_{L^{2}(\Omega)}^{\frac{1}{2 N}},
$$

where $C$ is independent of $i$ and $g$.

It follows from (53), (54) and (59) that (52) is proved for $\|g\|_{L^{2}(\Omega)} \leq 1$. Then there exists $\eta>0$ such that if $\|g\|_{L^{2}(\Omega)}<\eta$ then $\omega\left(\|g\|_{L^{2}(\Omega)}\right)<1$ and (52) implies that $\theta_{1}=\theta_{2}$ almost everywhere in $\Omega$.

The proof of Theorem 6.1 is complete.

Acknowledgement - The author thanks D. Blanchard and F. Murat for numerous discussions on the problems under investigation in the present paper.

\section{REFERENCES}

[1] R.A. Adams. Sobolev Spaces. Academic Press, 1975.

[2] P. Bénilan, L. Boccardo, T. Gallouët, R. Gariepy, M. Pierre, and J.L. Vazquez. An $L^{1}$-theory of existence and uniqueness of solutions of nonlinear elliptic equations. Ann. Scuola Norm. Sup. Pisa, 22:241-273, 1995.

[3] D. Blanchard and O. Guibé. Existence d'une solution pour un système non linéaire en thermoviscoélasticité. C.R. Acad. Sci. Paris Sér. I Math., 325:1125-1130, 1997.

[4] D. Blanchard and O. Guibé. Existence of a solution for a nonlinear system in thermoviscoelasticity. Adv. Differential Equations, 5(10-12):1221-1252, 2000.

[5] L. Boccardo, J.I. Diaz, D. Giachetti, and F. Murat. Existence and regularity of renormalized solutions for some elliptic problems involving derivations of nonlinear terms. $J$. Diff. Eq., 106:215-237, 1993.

[6] L. Boccardo and T. Gallouët. On some nonlinear elliptic and parabolic equations involving measure data. J. Funct. Anal., 87:149-169, 1989.

[7] B. Climent and E. Fernández-Cara. Existence an uniqueness results for a coupled problem related to the stationary Navier-Stokes. J. Math. Pures Appl., 76(4), 1997. 
[8] G. Dal Maso, F. Murat, L. Orsina, and A. Prignet. Definition and existence of renormalized solutions for elliptic equations with general measure data. C. R. Acad. Sci. Paris Sér. I Math., 325(5):481-486, 1997.

[9] G. Dal Maso, F. Murat, L. Orsina, and A. Prignet. Renormalized solutions of elliptic equations with general measure data. Ann. Scuola Norm. Sup. Pisa Cl. Sci. (4), 28(4):741-808, 1999.

[10] A. Dall'Aglio. Approximated solutions of equations with $L^{1}$ data. Application to the $H$ convergence of parabolic quasi-linear equations. Ann. Mat. Pura Appl.(4), 170:207-240, 1996.

[11] R.J. DiPerna and P.-L. Lions. On the Cauchy problem for Boltzmann equations : global existence and weak stability. Ann. of Math., 130(2):321-366, 1989.

[12] R.J. DiPerna and P.-L. Lions. Ordinary differential equations, Sobolev spaces and transport theory. Invent. Math., 98:511-547, 1989.

[13] T. Gallouët and R. Herbin. Existence of a solution to a coupled elliptic system. Appl. Math. Letters, 7:49-55, 1994.

[14] D. Gilbarg and N. S. Trudinger. Elliptic partial differential equations of second order. Springer-Verlag, 1977.

[15] O. Guibé. Existence de solutions pour des systèmes non linéaires couplés elliptiques ou d'évolution. $\mathrm{PhD}$ thesis, Université de Rouen, 1998.

[16] O. Guibé. Solutions entropiques et renormalisées pour un système non linéaire couplé. C. R. Acad. Sci. Paris Sér. I Math., 326(6):685-690, 1998.

[17] R. Lewandowski. Analyse mathématique et océanographie. Masson, Paris, 1997.

[18] P.-L. Lions and F. Murat. Solutions renormalisées d'équations elliptiques. (in preparation).

[19] F. Murat. Soluciones renormalizadas de EDP elipticas non lineales. Technical Report R93023, Laboratoire d'Analyse Numérique, Paris VI, 1993. Cours à l'Université de Séville.

[20] F. Murat. Equations elliptiques non linéaires avec second membre $L^{1}$ ou mesure. In Compte Rendus du 26ème Congrès d'Analyse Numérique, les Karellis, 1994. 